Chapter 19

\title{
Artery Bypass Versus PCI Using New Generation DES
}

\author{
Mohammed Balghith \\ Additional information is available at the end of the chapter \\ http://dx.doi.org/10.5772/54029
}

\section{Introduction}

Stents have substantially improved the safety and efficacy of percutaneous revascularization of atherosclerotic coronary arteries. The attendant risk of emergency referral for Coronary artery bypass graft surgery ( $C A B G$ ) and the need for subsequent revascularization procedures have been reduced by more than $50 \%$ since the use of new generation stents i.e drug eluted stents ( DES ) starting 2002. Comparisons of Percatouneous coronary intervention ( PCI ) and CABG have been made in 7 randomized trials designed to identify the most effective alternative for selected patients with multivessel CAD of whom both methods were deemed feasible. $[1,2]$. The individual results of these trials and a meta-analysis of their combined results have consistently shown equivalent survival rates with use of the 2 strategies over approximately 5 years of follow-up.

\section{Coronary artery bypass graft surgery}

A coronary artery bypass surgery is a surgical procedure performed to relieve angina and reduce the risk of death from coronary artery disease. Arteries or veins from elsewhere in the patient's body are grafted to the coronary arteries to bypass atherosclerotic narrowings and improve the blood supply to the coronary circulation supplying the myocardium (heart muscle). Example, Left internal mammary artery (LIMA) graft to LAD and SVG to OM and RCA (figure 1). The operation is usually performed with the heart stopped, requiring the usage of cardiopulmonary bypass; other methods are available to achieve CABG on a beating heart, so-called "off-pump" surgery. [3]. 


\section{Advantages of CABG}

Over the last 4 decades, surgical coronary artery revascularization techniques and technology have advanced significantly. As a result, despite an increasingly older and sicker patient population, CABG outcomes continue to improve. For example, the predicted mortality of CABG patients has increased steadily over the past decade, yet observed operative mortality rates have decreased, [4]. This is partly because advances in preoperative evaluation, including more precise coronary artery and myocardial imaging and diagnostic techniques, have allowed more appropriate patient selection and surgical planning. In addition, preoperative, intraoperative, and postoperative monitoring and therapeutic interventions have made CABG safer, even for critically ill and high-risk patients. Improvements in cardiopulmonary perfusion and careful myocardial protection, as well as the use of off-pump and on-pump beatingheart techniques in selected patients, have also decreased perioperative morbidity and mortality rates. $[5,6]$.

Use of the bilateral IMAs offers the possibility of constructing various configurations, making total arterial myocardial revascularisation possible with a minimum number of arterial conduits. Use of the skeletonised RIMA through the transverse sinus and eventually retrocavally can reach most branches of the circumflex system and is associated with an excellent patency rate. Patients who received bilateral IMA grafts for left coronary system revascularisation had improved early and late outcomes and decreased risk of death, reoperation, and angioplasty. [7].

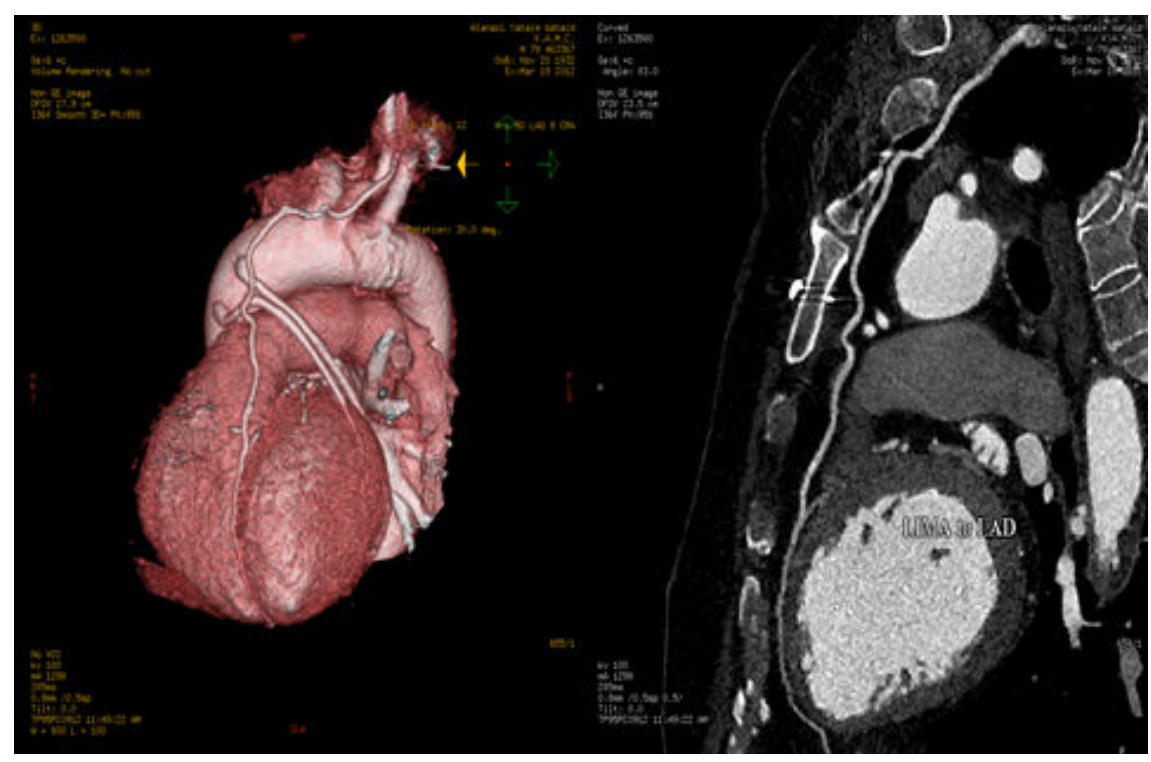

Figure 1. CT coronary angiogram, showing a CABG done 5 years ago with LIMA to LAD artery and SVG to OM and RCA. 


\section{Percutaneous coronary intervention using drug eluted stents}

Percutaneous coronary intervention (PCI) involves dilatation of an obstructed or narrowed coronary artery, using a balloon catheter to dilate the artery from within. After balloon dilatation, a stainless steel stent is usually placed in the coronary artery. Antiplatelet agents like aspirin or clopidogrel are mandatory to be used after stenting. Stents may be either bare metal (BMS) or drug-eluting stents (DES). Indications for PCI might be elective or emergency according to the clinical presentations of the patients. Primary PCI in the setting of ST segment elevation myocardial infarction (STEMI): When the catheterization lab including the team and facility is available, angioplasty with stenting is the optimal method of reperfusion for STEMI. The target "door to balloon time" is 90 minutes, [8]. Rescue PCI is considered as a treatment in patients with thrombolysis - if there is failure to reperfuse, further ischaemia with persistant chest pain, or continuous ST elevation. PCI is considered also as an early invasive strategy in Acute coronary syndrome, Non-ST elevation myocardial infarction (NSTEMI) and unstable angina: [9]., or conservative strategy for patients who are at medium-to-high risk of subsequent cardiac events. Elective PCI for patient with Stable angina or positive stress test: with single or double vessel disease, where optimal medical therapy fails to control symptoms. Patients with triple vessel disease, who are unsuitable for CABG, [10].

\section{The Cypher ${ }^{\text {тм }}$ Sirolimus-Coated Stent}

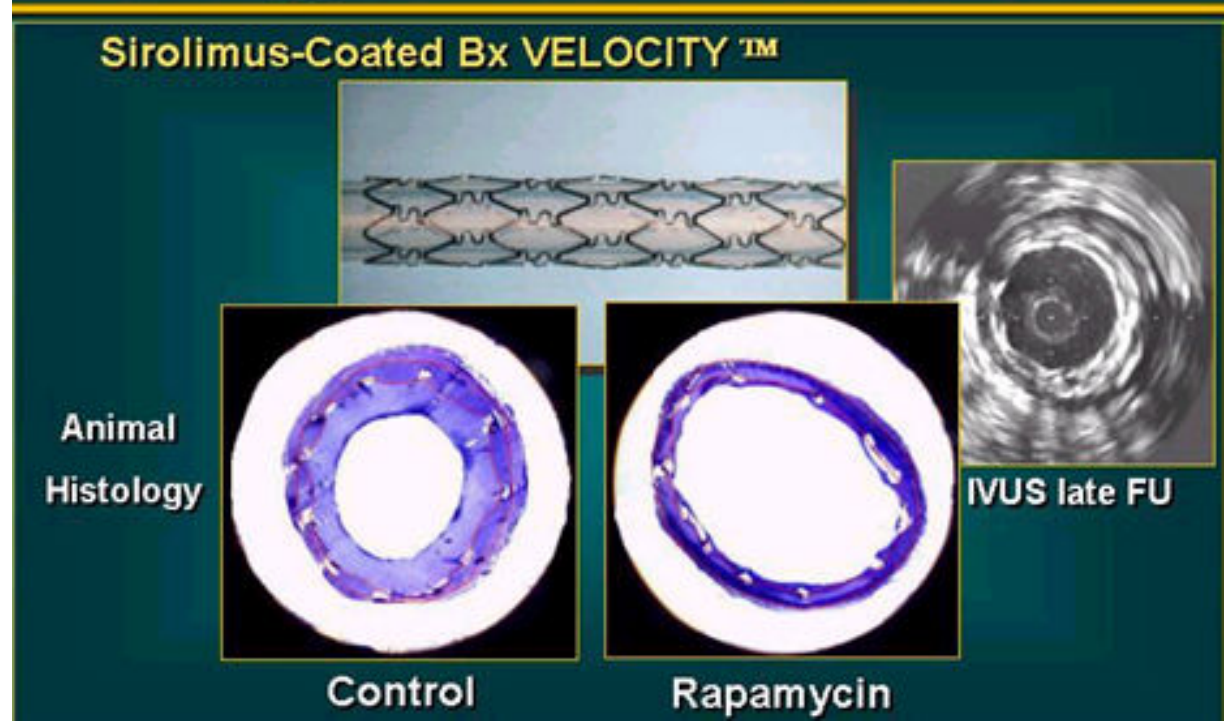

Figure 2. Cypher Stent- Siroliums eluted stent 
A drug-eluting stent presents or releases single or multiple bioactive agents into the blood stream. The drug can deposit in and/or affect blood vessels, cells, plaque, or tissues either adjacent to the stent or at a distance. The drug can be embedded and released from within ("matrix-type") or surrounded by and released through ("reservoir-type") polymer materials that coat ("strut-adherent") or span ("strut-spanning") the struts of the stents. These agents prevent in-stent restenosis by reducing the intimal hyperplasia, [11].

The advantages and a lower cost compared to CABG makes DES an attractive option to treat coronary artery disease. Currently, five DESs are available in the USA: the CYPHER sirolimuseluting stent from Cordis (approved by FDA on 24 April 2003), Figure 2, 3. The TAXUS Express and Liberté paclitaxel-eluting stents from Boston Scientific (approved by FDA on 4 March 2004 and 10 October 2008, respectively) (TAXUS Express is referred to as TAXUS) Figure 4, the ENDEAVOR zotarolimus-eluting stent from Medtronic (approved by FDA on 1 February 2008), and the XIENCE V Figure 5, everolimus-eluting stent from Abbott Vascular (approved by FDA on 2 July 2008). [12].
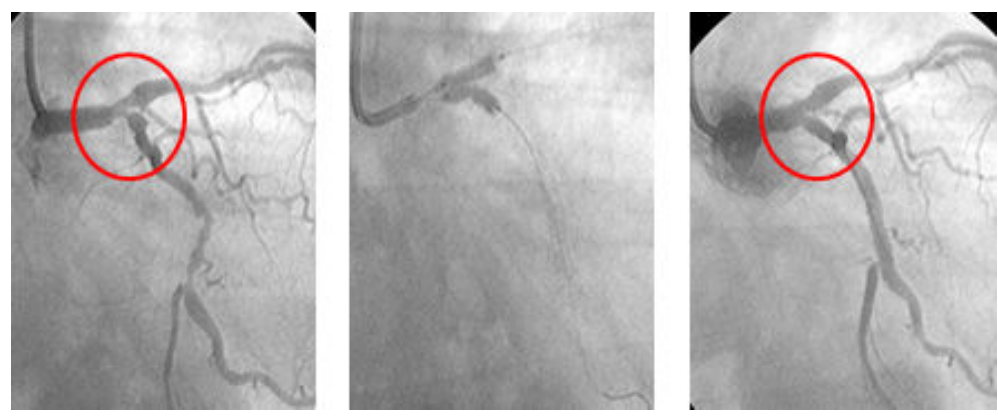

Figure 3. Complex case with LM disease treated by 2 Cypher stents

\section{TAXUS Stent--Paclitaxal Coated Stent}

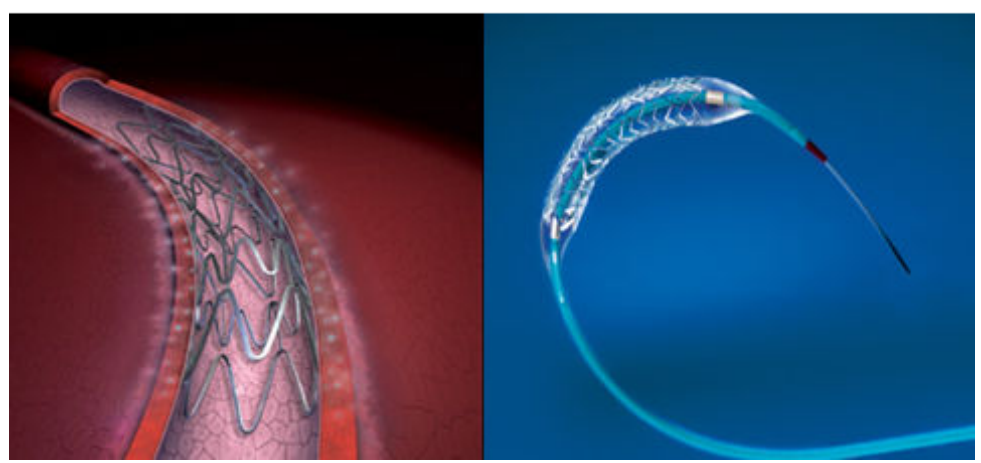

Figure 4. Taxus stent-Paclitaxal eluted stent 


\section{RCA 99\% stenosis treated by Xience stent}

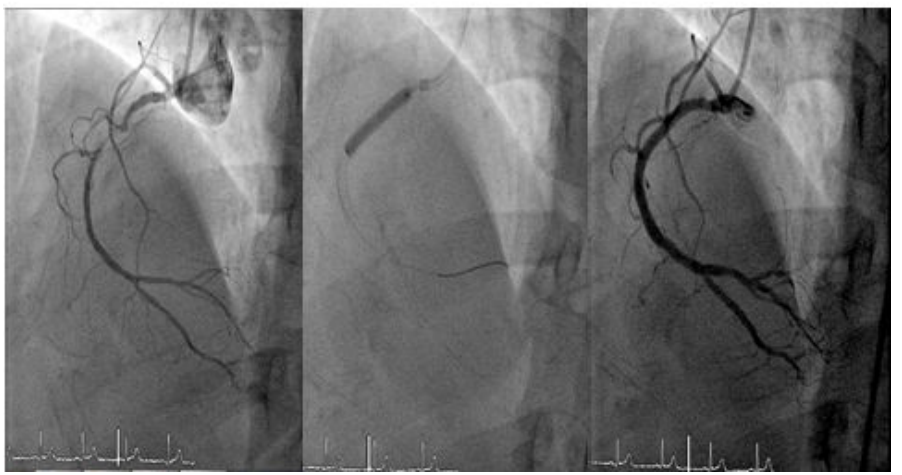

Figure 5. Significan disease of proximal RCA treated by Xience stent

\section{Outcomes of coronary-artery bypass grafting versus bare metal stent implantation}

The New York's cardiac registries were one of the largest studies which identify 37,212 patients with multivessel disease who underwent CABG and 22,102 patients with multivessel disease who underwent PCI using BMS from January 1, 1997, to December 31, 2000. They determined the rates of death and subsequent revascularization within three years after the procedure in various groups of patients according to the number of diseased vessels and presence or absence of involvement of the left anterior descending coronary artery LAD.

Risk-adjusted survival rates were significantly higher among patients who underwent CABG than among those who received a stent in all of the anatomical subgroups studied. For example, the adjusted hazard ratio for the long-term risk of death after CABG relative to stent implantation was 0.64 (95 percent confidence interval, 0.56 to 0.74 ) for patients with three-vessel disease with involvement of the proximal LAD and 0.76 (95 percent confidence interval, 0.60 to 0.96 ) for patients with two-vessel disease with involvement of the non-proximal LAD. Also, the three-year rates of revascularization were considerably higher in the stenting group than in the CABG group (7.8 percent vs. 0.3 percent for subsequent CABG and 27.3 percent vs. 4.6 percent for subsequent PCI), [13].

Texas Heart Institute Cardiovascular Research Database retrospectively identified patients who had undergone their 1 st revascularization procedure with coronary artery bypass surgery (CABG; $n=2,826)$ or coronary stenting $(n=2,793)$ between January 1995 and December 1999. They have found that in-hospital mortality was significantly greater in patients undergoing CABG than in those undergoing stenting ( $3.6 \%$ vs $0.75 \%$; adjusted OR $8.4 ; P<0.0001$ ). At a mean 2.5-year follow-up, risk-adjusted survival was equivalent (CABG 91\%, stenting 95\%; 
adjusted OR 1.26; $P=0.06$ ). When subgroups matched for severity of disease were compared, no differences in risk-adjusted survival were seen, [14].

\section{Drug-eluting stents vs coronary artery bypass surgery for the treatment of multivessel coronary disease}

A Chinese study identified 3720 consecutive patients with multivessel disease who underwent isolated CABG surgery or received drug-eluting stents between April 1, 2004, and December 31, 2005, which compared safety (total mortality, myocardial infarction, and stroke) and efficacy (target-vessel revascularization) during a 3-year follow-up. These outcomes were compared after adjustment for the differences in baseline risk factors. Patients who underwent CABG $(\mathrm{n}=1886)$ were older and had more comorbidities than patients who received drug-eluting stents $(n=1834)$. Patients receiving drug-eluting stents had considerably higher 3-year rates of target-vessel revascularization. Drug-eluting stents were also associated with higher rates of death (adjusted hazard ratio, 1.62; 95\% confidence interval, 1.07 to 2.47 ) and myocardial infarction (adjusted hazard ratio, 1.65; $95 \%$ confidence interval, 1.15 to 2.44 ). The risk adjusted rate of stroke was similar in the 2 groups (hazard ratio, $0.92 ; 95 \%$ confidence interval, 0.69 to 1.51 ). [15]

In a Korean study, a 5-year clinical follow-up of 395 patients with unprotected LMCA disease who underwent PCI with drug-eluting stents (DES) $(n=176)$ or CABG $(n=219)$ was preformed from January 2003 to May 2004. In the 5-year follow-up, cohort of DES and concurrent CABG, there had not been a significant difference in the adjusted risk of death (HR: 0.83; 95\% CI: 0.34 to $2.07 ; \mathrm{p}=0.70$ ) or the risk of the composite outcome (HR: $0.91 ; 95 \% \mathrm{CI}: 0.45$ to $1.83 ; \mathrm{p}=0.79$ ). The rates of TVR were also higher in the DES group than the CABG group (HR: 6.22; 95\% CI: 2.26 to $17.14 ; \mathrm{p}<0.001)$, [16].

In an Italian study, 249 patients: 107 of whom were treated with PCI along with DES implantation and 142 treated with CABG. At 5-year clinical follow-up, no difference was found between PCI and CABG in the occurrence of cardiac death (adjusted odds ratio [OR]: 0.502; 95\% confidence interval [CI]: 0.162 to $1.461 ; \mathrm{p}=0.24)$. The PCI group showed a trend toward a lower occurrence of the composite end point of cardiac death and MI (adjusted OR: 0.408 ; 95\% CI: 0.146 to $1.061 ; \mathrm{p}=0.06$ ). Percutaneous coronary intervention was associated with a lower rate of the composite end point of death, MI, and/or stroke (OR: 0.399; 95\% CI: 0.151 to 0.989; $\mathrm{p}=0.04$ ). Indeed, CABG was correlated with lower target vessel revascularization (adjusted OR: 4.411; 95\% CI: 1.825 to $11.371 ; \mathrm{p}=0.0004$ ). No difference was detected in the occurrence of major adverse cardiac and cerebrovascular events (adjusted OR: 1.578; 95\% CI: 0.825 to 3.054; p = 0.18) [17].

In a Meta-analysis of clinical studies comparing CABG with DES in patients with unprotected left main coronary artery narrowing, the analysis included 2,905 patients from 8 clinical studies (2 randomized trials and 6 nonrandomized studies). At 1-year follow-up, there was no significant difference between the CABG and DES groups in the risk for death (odds ratio [OR] 1.12, 95\% confidence interval [CI] 0.80 to 1.56) or the composite 
end point of death, myocardial infarction, or stroke (OR 1.25, 95\% CI 0.86 to 1.82). The risk for target vessel revascularization was significantly lower in the CABG group compared to the PCI group (OR 0.44, 95\% CI 0.32 to 0.59). In conclusion, PCI with DES is safe and could represent a good alternative to CABG for selected cases in patients with ULMCA disease, [18].

In the SYNTAX trial, 1,800 patients with three-vessel and/or LM disease were randomized to either CABG or PCI; of these, 271 LM patients were prospectively assigned to receive a 15-month angiogram. The primary endpoint for the CABG arm was the ratio of $\geq 50 \%$ to $<100 \%$ obstructed/occluded grafts bypassing LM lesions to the number placed. The primary endpoint for the PCI arm was the proportion of patients with $\leq 50 \%$ diameter stenosis ('patent' stents) of treated LM lesions. Per protocol, no formal comparison between CABG and PCI arms was intended based on the differing primary endpoints. Available 15-month angiograms were analyzed for 114 CABG and 149 PCI patients. At 15 months, 9.9\% (26/263) of CABG grafts were 100\% occluded and an additional $5.7 \%$ (15/263) were $\geq 50 \%$ to $<100 \%$ occluded. Overall, $27.2 \%$ (31/114) of patients had $\geq 1$ obstructed/occluded graft. The 15-month CABG MACCE rate was $8.8 \%(10 / 114)$ and MACCE at 15 months was not significantly associated with graft obstruction/occlusion $(\mathrm{p}=0.85)$. In the PCI arm, $92.4 \%(134 / 145)$ of patients had $\leq 50 \%$ diameter LM stenosis at 15 months (89.7\% [87/97] distal LM lesions and 97.9\% [47/48] non-distal LM lesions). The 15-month PCI MACCE rate was $12.8 \%$ (20/156) and this was significantly associated with lack of stent patency at 15 months $(\mathrm{p}<0.001)$, mainly due to repeated revascularization. [19].

The results of the SYNTAX trial confirm that at 3 years CABG remains the treatment of choice for most patients with three-vessel and LMS disease and especially in those with the most severe disease. SYNTAX will have a profound effect on practice recommendations for the foreseeable future and has already had a major effect on the new European Society for Cardiology/European Association for Cardiothoracic Surgery guidelines for myocardial revascularization, [20].

At four years follow-up of SYNTAX trial which presented at TCT in 2011, there was no difference in MACCE between CABG and PCI in those with a SYNTAX score of 0 to $22,(26.1 \%$ vs $28.6 \%$; $=0.57$ ). This is good, and would legitimize the use of PCI in this kind of patient". But for those with an intermediate SYNTAX score of 23 to 32, "You see immediately a highly significant difference" in MACCE rate (21.5\% for CABG vs $32 \%$ for PCI; $\mathrm{p}=0.006)$. For those with a high SYNTAX score ( $\geq 33)$, "mortality is double in the PCI group compared with CABG $(16.1 \%$ vs $8.4 \%$; $\mathrm{p}=0.04)$ in addition to $\mathrm{MI}$ is two to three times higher with PCI than with CABG $(9.3 \%$ vs $3.9 \%$; $=0.01)$.

In this highest-risk group, even the end point of death/stroke/MI becomes significantly higher with PCI, (22.7\% vs $14.6 \%$; $=0.01)$, and MACCE were much higher ( $40.1 \%$ vs $23.6 \%$; $<<0.001)$, driven in large part by a $17 \%$ higher rate of revascularization in this high-risk group at four years. Figures $6 \& 7$ 


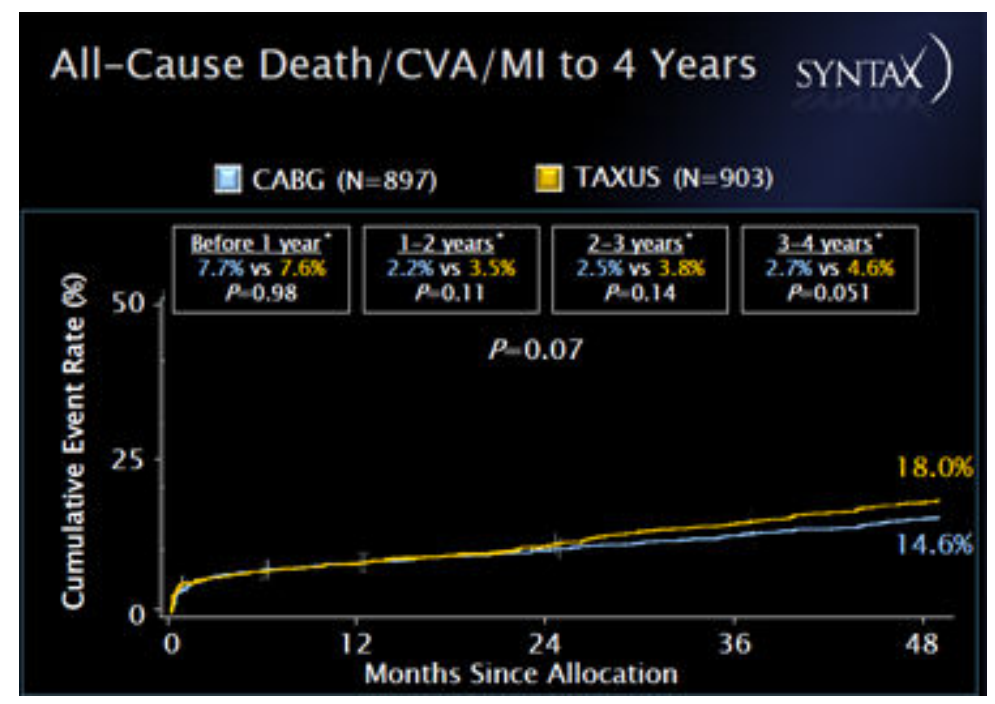

Figure 6. years follow up in Syntax study, demonstrate all cause death/CVA/MI up to 4 years

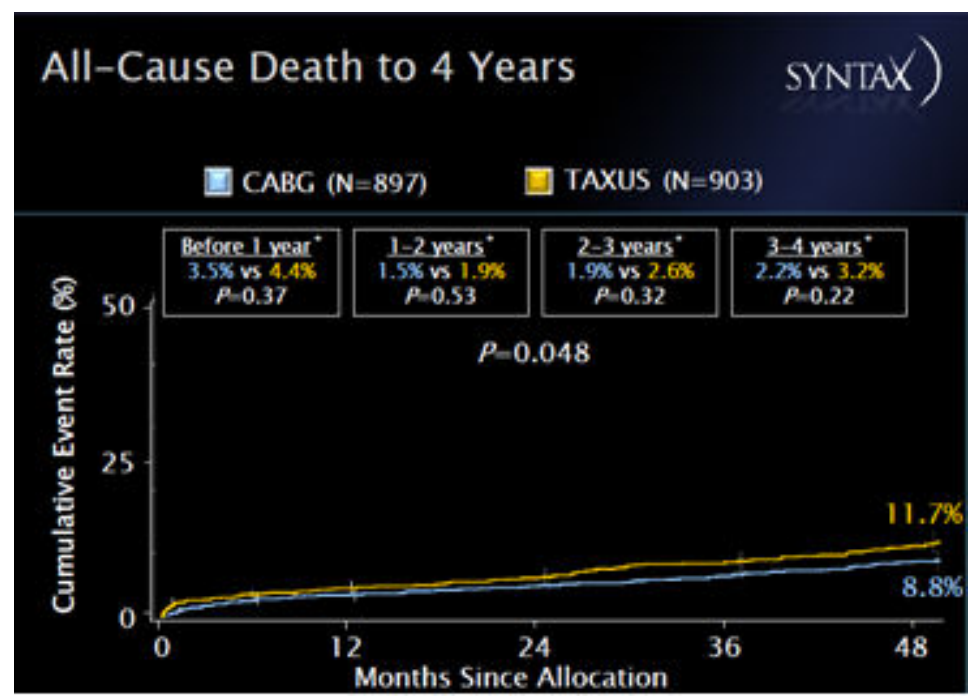

Figure 7. years follow up in Syntax study, demonstrate all cause death up to 4 years 


\section{Revascularization for patients with diabetes mellitus and multivessel CAD}

In the BARI 2D trial, the selected revascularization strategy, CABG or PCI, was based on physician discretion, declared independent of randomization to either immediate or deferred revascularization if clinically warranted. They analyzed factors favoring selection of CABG versus PCI in 1,593 diabetic patients with multivessel CAD enrolled between 2001 and 2005. The majority of diabetic patients with multivessel disease were selected for PCI rather than CABG. Preference for CABG over PCI was largely based on angiographic features related to the extent, location, and nature of $\mathrm{CAD}$, as well as geographic, demographic, and clinical factors. [21]

However, with each intervention the benefit is less and the risks and complications are greater than in patients without diabetes. Revascularization for treatment of ST elevation myocardial infarction increases survival. Both interventions relieve symptoms, but neither improves survival except in patients at high risk. In patients with clinically stable chronic coronary disease, survival after CABG or PCI is comparable with that in patients treated with optimal medical therapy alone. Accordingly, evaluation for revascularization can be deferred until signs and symptoms worsen except in patients at high risk. In patients at high risk survival after promptly implemented CABG is greater than that with optimal medical therapy, especially when the diabetes is being treated with insulin sensitizing agents. [22]

\section{Quality of life after PCI with DES or CABG}

Among patients with three-vessel or left main coronary artery disease who were suitable candidates for either PCI using DES or CABG, both strategies resulted in significant relief from angina and improvements in overall health status over the first year of follow-up. At both 6 and 12 months, there was a small but significant reduction in angina frequency with CABG as compared with PCI in the overall population. These symptomatic benefits of CABG were counterbalanced by the more rapid recovery and improved short-term health status achieved with PCI. [23]

\section{Future study with the second generation des and other bioabsorbable stents}

EXCEL is a 2600-patient study comparing patients with left main disease randomized to bypass surgery or PCI with the Xience stent and followed for at least three years. The primary end point is death, stroke, and MI; repeat revascularization is a secondary end point. EXCEL results awaited. Figure (8) 


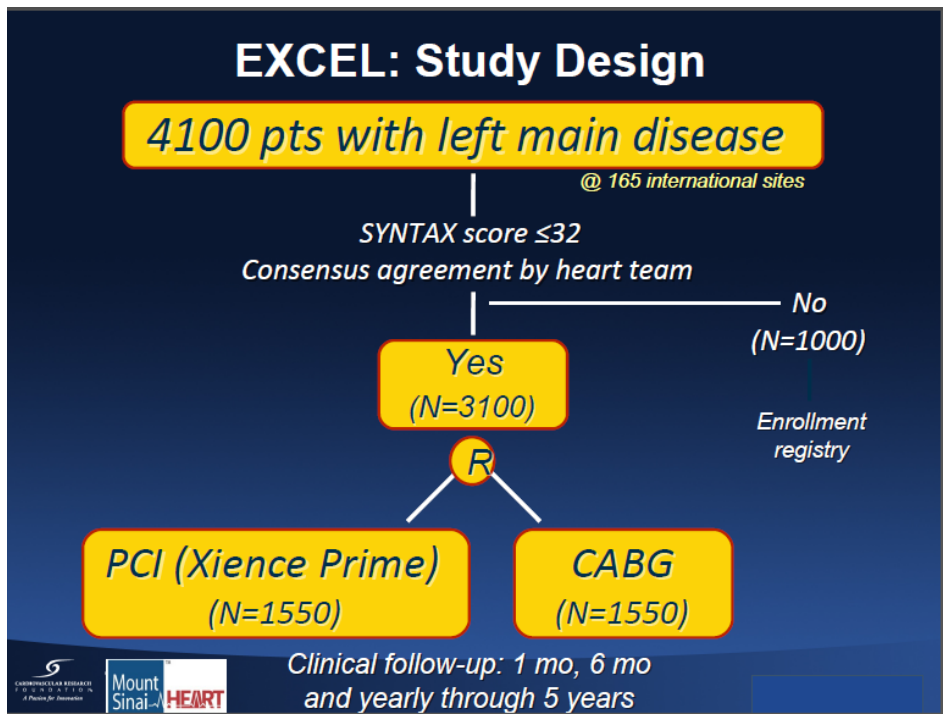

Figure 8. EXCEL study protocol comparing Xience stent with CABG

\section{The BVS everolimus-eluting stent system}

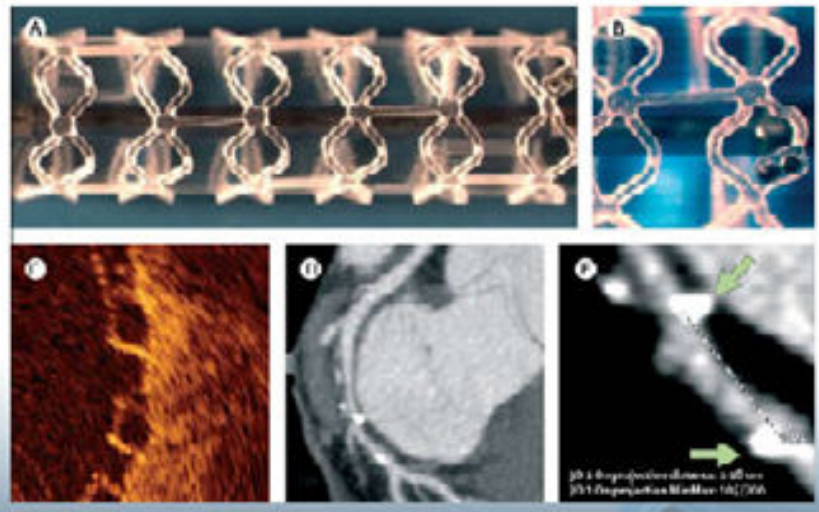

Figure 9. Absorb Stent- Bioabsorbale everolimus eluted stent

Stents composed of bioabsorbable/biodegradable materials represent an attractive alternative revascularization modality; the justification stems from the short-term need for vessel scaffolding and avoidance of the potential long-term complications of metallic stents. Compared with 
metallic stents, there are several potential advantages, including complete absorption of stent material, [24], Abbott Vascular ABSORB Everolimus Eluting Bioresorbable Vascular Scaffold System"These outcomes suggest that a temporary scaffold like ABSORB provides durable results over the long term and a permanent implant may not be necessary to effectively treat patients with coronary artery disease". ABSORB II trial is ongoing. Figure (9)

\section{Combining the best of both worlds hybrid coronary revascularization}

As PCI technology improves and techniques of LIMA-to-LAD grafting become less invasive, hybrid coronary revascularization is becoming a distinct possibility. For example, a minimally invasive, off-pump, direct LIMA-to-LAD anastomosis can be combined with DES placement in a focal mid-right-coronary-artery lesion in a patient with complex proximal LAD lesions. Hybrid coronary revascularization procedures are currently being performed, with promising early results. A few centers, now have hybrid operating rooms with cardiac surgical and coronary angiographic capabilities that make it possible to perform simultaneous hybrid coronary revascularizations. Staged hybrid revascularizations are performed in standard catheterization laboratories and operating rooms. [25,26].

\section{Conclusion}

Each strategy can have great outcomes in appropriately selected patients. Hard clinical outcomes (death/MI/CVA) are generally similar, need to weigh the risk of potential repetition of procedures with PCI using DES vs. the greater morbidity of CABG. The 3VD and LMCA Disease are high-risk coronary lesions and the least stable subtypes of "stable CAD" PCI and CABG have very similar rates of hard clinical endpoints. Greater rates of recurrent revascularization with PCI, especially in complex disease, Patient selection and patient preference will generally dictate the best and most appropriate care. The so-called SYNTAX score, evolved for the trial, offers a grading system, based on patient anatomy, to help surgeons and interventionalists make this decision. As PCI and CABG are refined further, surgeons and cardiologists will no doubt learn to use these improved interventional techniques and surgical procedures in a way that will optimize the treatment of each individual patient.

\section{Author details}

Mohammed Balghith

King Saud Bin Abdulaziz University for Heath Sceinces, KACC, National Guard, Riyadh, Saudi Arabia 


\section{References}

[1] Rodriguez A, Boullon F, Perez-Balino N, Paviotti C, Liprandi MI, Palacios IF. Argentine randomized trial of percutaneous transluminal coronary angioplasty versus coronary artery bypass surgery in multivessel disease (ERACI): in-hospital results and 1-year follow-up. ERACI Group. J Am Coll Cardiol 1993;22:1060-7.

[2] Hamm CW, Reimers J, Ischinger T, Rupprecht HJ, Berger J, Bleifeld W. A randomized study of coronary angioplasty compared with bypass surgery in patients with symptomatic multivessel coronary disease. German Angioplasty Bypass Surgery Investigation (GABI). N Engl J Med 1994;331: 1037-43

[3] Kolessov et al, "Mammary artery-coronary artery anastomosis as method of treatment for angina pectoris". J Thorac Cardiovasc Surg VI (October 1967). 54 (4): 535-44

[4] Ferguson TB Jr, Hammill BG, Peterson ED, DeLong ER, Grover FL, STS National Database Committee. A decade of change: risk profiles and outcomes for isolated coronary artery bypass grafting procedures, 1990-1999: a report from the STS National Database Committee and the Duke Clinical Research Institute. Society of Thoracic Surgeons. Ann Thorac Surg 2002;73:480-9.

[5] Al-Ruzzeh S, Ambler G, Asimakopoulos G, Omar RZ, Hasan R, Fabri B, et al. Offpump coronary artery bypass (OPCAB) surgery reduces risk-stratified morbidity and mortality: a United Kingdom multi-center comparative analysis of early clinical outcome. Circulation 2003;108 Suppl 1:II1-8.

[6] Puskas JD, Williams WH, Mahoney EM, Huber PR, Block PC, Duke PG, et al. Offpump vs conventional coronary artery bypass grafting: early and 1-year graft patency, cost, and quality-of-life outcomes: a randomized trial. JAMA 2004;291:1841-9.

[7] M Bonacchi, Battaglia F, Prifti E, Leacche M, Nathan N S, Sani G, and Popoff G,Early and late outcome of skeletonised bilateral internal mammary arteries anastomosed to the left coronary system Heart. 2005 February; 91(2): 195-202. Ratcliffe A T, C Pepper. Thrombolysis or primary angioplasty? Reperfusion therapy for myocardial infarction in the UK, Postgrad Med J2008;84:73-77

[8] Ratcliffe A T , C Pepper . Thrombolysis or primary angioplasty? Reperfusion therapy for myocardial infarction in the UK, Postgrad Med J2008;84:73-77

[9] McCullough PA, Gibson CM, Dibattiste PM, et al. Timing of angiography and revascularization in acute coronary syndromes: an analysis of the TACTICS-TIMI-18 trial. J Interv Cardiol 2004;17:81-6

[10] Levine GN, Bates ER, Blankenship JC, et al. 2011 ACCF/AHA/SCAI guideline for percutaneous coronary intervention: A report of the American College of Cardiology Foundation/American Heart Association Task Force on Practice Guidelines and the 
Society for Cardiovascular Angiography and Interventions. J Am Coll Cardiol 2011;08.007

[11] Robert S. Schwartz, MD; Elazer R. Edelman, MD, PhD; Andrew Carter, DO; Nicolas Chronos, MD; Campbell Rogers, MD; Keith A. Robinson, PhD; Ron Waksman, MD; Judah Weinberger, MD; Robert L. Wilensky, MD; Donald N. Jensen, DVM; Bram D. Zuckerman, MD; Renu VirmaniDrug-Eluting Stents in Preclinical Studies, Recommended Evaluation From a Consensus Group, Circulation. 2002; 106: 1867-1873

[12] Doostzadeh J, Clark LN, Bezenek S, Pierson W, Sood PR, Sudhir K. Recent progress in percutaneous coronary intervention: evolution of the drug-eluting stents, focus on the XIENCE V drug-eluting stent. Coron Artery Dis. 2010 Jan;21(1):46-56.

[13] Hannan EL, Racz MJ, Walford G, Jones RH, Ryan TJ, Bennett E, Culliford AT, Isom OW, Gold JP, Rose EA, N Engl J Med. Long-term outcomes of coronary-artery bypass grafting versus stent implantation. 2005 May 26;352(21):2174-83.

[14] Rollo P. Villareal, MD, Vei-Vei Lee, MS, MacArthur A. Elayda, MD, PhD, and James M. Wilson, MD Coronary Artery Bypass Surgery versus Coronary Stenting, Risk-Adjusted Survival Rates in 5,619 Patients. Tex Heart Inst J. 2002; 29(1): 3-9

[15] Li Y, Zheng Z, Xu B, Zhang S, Li W, Gao R, Hu S.Comparison of drug-eluting stents and coronary artery bypass surgery for the treatment of multivessel coronary disease: three-year follow-up results from a single institution. Circulation. 2009 Apr 21;119(15):2040-50

[16] Park DW, Kim YH, Yun SC, Lee JY, Kim WJ, Kang SJ, Lee SW, Lee CW, Kim JJ, Choo SJ, Chung CH, Lee JW, Park SW, Park SJ. Long-term outcomes after stenting versus coronary artery bypass grafting for unprotected left main coronary artery disease: 10year results of bare-metal stents and 5-year results of drug-eluting stents from the ASAN-MAIN (ASAN Medical Center-Left MAIN Revascularization) Registry. J Am Coll Cardiol. 2010 Oct 19;56(17):1366-75.

[17] Chieffo A, Magni V, Latib A, Maisano F, Ielasi A, Montorfano M, Carlino M, Godino C, Ferraro M, Calori G, Alfieri O, Colombo A. 5-year outcomes following percutaneous coronary intervention with drug-eluting stent implantation versus coronary artery bypass graft for unprotected left main coronary artery lesions the Milan experience. JACC Cardiovasc Interv. 2010 Jun;3(6):595-601.

[18] Lee MS, Yang T, Dhoot J, Liao H. Meta-analysis of clinical studies comparing coronary artery bypass grafting with percutaneous coronary intervention and drug-eluting stents in patients with unprotected left main coronary artery narrowings. Am J Cardiol. 2010 Apr 15;105(8).

[19] Morice MC, Feldman TE, Mack MJ, Ståhle E, Holmes DR, Colombo A, Morel MA, van den Brand M, Serruys PW, Mohr F, Carrié D, Fournial G, James S, Leadley K, Dawkins KD, Kappetein AP. Angiographic outcomes following stenting or coronary artery bypass surgery of the left main coronary artery: fifteen-month outcomes from 
the synergy between PCI with TAXUS express and cardiac surgery left main angiographic substudy (SYNTAX-LE MANS). EuroIntervention. 2011 Oct 30;7(6):670-9.

[20] Taggart DP. Lessons learned from the SYNTAX trial for multivessel and left main stem coronary artery disease. Curr Opin Cardiol. 2011 Nov;26(6):502-7.

[21] Kim LJ, King SB 3rd, Kent K, Brooks MM et al; BARI 2D (Bypass Angioplasty Revascularization Investigation Type 2 Diabetes) Study Group.Factors related to the selection of surgical versus percutaneous revascularization in diabetic patients with multivessel coronary artery disease in the BARI 2D (Bypass Angioplasty Revascularization Investigation in Type 2 Diabetes) trial. JACC Cardiovasc Interv. 2009 May; 2(5):384-92.

[22] Sobel BE.Coronary revascularization in patients with type 2 diabetes and results of the BARI 2D trial. Coron Artery Dis. 2010 May;21(3):189-98.

[23] David J. Cohen, M.D., Ben Van Hout, Ph.D., Patrick W. Serruys, M.D., Ph.D., for the Synergy between PCI with Taxus and Cardiac Surgery (SYNTAX) Investigators, Quality of Life after PCI with Drug-Eluting Stents or Coronary-Artery Bypass Surgery. N Engl J Med 2011; 364:1016-1026March 17, 2011

[24] Waksman R, Erbel R, Di Mario C, et al; PROGRESS-AMS (Clinical Performance Angiographic Results of Coronary Stenting with Absorbable Metal Stents) Investigators. Early- and long-term intravascular ultrasound and angiographic findings after bioabsorbable magnesium stent implantation in human coronary arteries. JACC Cardiovasc Intervent. 2009;2:312-320

[25] de Canniere D, Jansens JL, Goldschmidt-Clermont P, Barvais L, Decroly P, Stoupel E. Combination of minimally invasive coronary bypass and percutaneous transluminal coronary angioplasty in the treatment of double-vessel coronary disease: two-year follow-up of a new hybrid procedure compared with "on-pump" double bypass grafting. Am Heart J 2001;142:563-70.

[26] Lee MS, Wilentz JR, Makkar RR, Singh V, Nero T, Swistel D, et al. Hybrid revascularization using percutaneous coronary intervention and robotically assisted minimally invasive direct coronary artery bypass surgery. J Invasive Cardiol 2004;16:419-25. 